\title{
Evaluation of skeletal muscle mass as a predictor of prognosis in patients treated in hospital for COVID-19 infection
}

\author{
SAHIN Cem ${ }^{1}$, YENICERI Ibrahim Onder ${ }^{2}$, ORAL TAPAN Ozge ${ }^{3}$, CAKIR Tumay $^{4}$, \\ DIRGEN CAYLAK Selmin ${ }^{5}$, TOGAN Turhan ${ }^{5}$
}

Department of Chest Diseases, Mugla Sitki Kocman University, Mugla, Turkey. ozgeeoral@hotmail.com

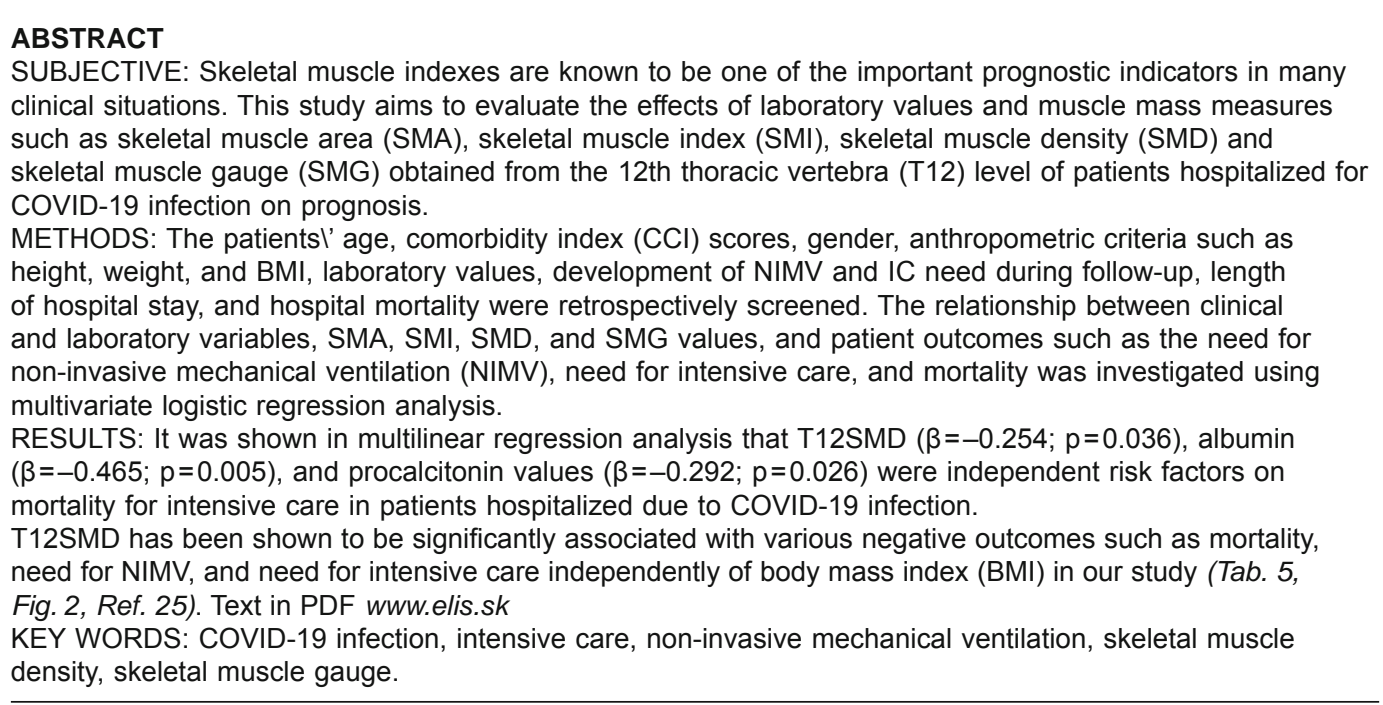

\section{Introduction}

Coronaviruses $(\mathrm{CoV})$ are a large family of viruses that can cause more serious infections such as Middle East Respiratory Syndrome (MERS) and Severe Acute Respiratory Syndrome (SARS) from self-limiting mild infections that are common in society such as colds (1). The disease spectrum ranges from a simple cold to severe acute respiratory syndrome. Common symptoms of infection are respiratory symptoms, fever, cough, and dyspnea. Pneumonia, severe acute respiratory tract infection, renal failure, and even death may develop in more serious cases.

\footnotetext{
${ }^{1}$ Department of Internal Medicine, Mugla Sitki Kocman University, Mugla, Turkey, ${ }^{2}$ Department of Radiology, Mugla Sitki Kocman University, Mugla, Turkey, ${ }^{3}$ Department of Chest Diseases, Mugla Sitki Kocman University, Mugla, Turkey, ${ }^{4}$ Department of Intensive Care, Mugla Training and Research Hospital, Mugla, Turkey, and ${ }^{5}$ Department of Infectious Diseases, Mugla Sitki Kocman University, Mugla, Turkey

Address for correspondence: O.O. Tapan, Department of Chest Diseases, Mugla Sitki Kocman University, Kotekli Mah. Marmaris Cad. Mugla, 48000, Turkey.

Phone: +902522115244
}

Thoracic CT provides valuable information about the diagnosis, disease severity, and patient prognosis although the detection of viral RNA in nasopharyngeal swabs or tracheal aspirates by reverse transcription-polymerase chain reaction (RT-PCR) is the gold standard for diagnosis (2). Thoracic CT is also used as an early sensitive diagnostic approach in COVID-19 patients with negative PCR testing. Several studies have shown that thoracic CT can be useful as a standard method to optimize the rapid diagnosis and management of COVID-19 due to the low rate of missed diagnosis of COVID-19 (3.9\%) (3).

Sarcopenia, which is defined as progressive generalized loss of muscle mass, muscle strength, and function, is a clinical syndrome that may accompany many diseases with chronic and acute inflammation (4). Low muscle area and indices are known to be one of the poor prognostic factors in many benign and malignant diseases. Weight loss and loss of muscle mass are common in COVID-19 infection due to the effect of anosmia and loss of taste as well as high inflammatory cytokine levels. Body mass index (BMI) is an inadequate criterion in distinguishing muscle and fat tissue even though it is one of the most common measures of body size. Low BMI may mask excessive adiposity whereas high BMI may mask sarcopenia. Therefore, evaluation of body composition criteria that 


\section{7-204}

can distinguish body fat tissue distribution, muscle quantity, and quality may be more useful in evaluating sarcopenia.

Various tools such as Dual-energy X-ray absorptiometry (DXA), bioelectric impedance analysis (BIA), ultrasound (US), $\mathrm{CT}$, and MRI can be used to determine muscle mass (5). The measurement of muscle mass at a vertebral level in patients hospitalized for COVID-19 infection appears to be one of the best options. Skeletal muscle measurements at the level of the third lumbar (L3) vertebra are generally used for this purpose. However, it is reported in the literature that other thoracic segments can be used as an alternative in cases where L1, L2, L3, L4, L5, and 10th, 11th and 12th thoracic (T12) vertebra measurements do not fall within the L3 segment area due to their strong correlation (6-8).

Frequently used criteria for the measurement of muscle mass at a vertebral level in CT are SMA - Skeletal muscle area, SMI Skeletal muscle index, and SMD - Skeletal muscle density. Skeletal muscle index (muscle area in $\mathrm{cm}^{2}$ at SMI - L3) and skeletal muscle density (SMD) measurements were frequently used in studies conducted to evaluate sarcopenia with CT imaging. However, it has been suggested to use SMG measurement obtained by multiplying SMI and SMD measurements as a new marker in the differentiation of sarcopenia since muscle density and quantity do not always show a positive correlation, considering that the use of a mathematical combination showing both muscle quantity (SMI) and density (SMD) may give more meaningful results in terms of predicting sarcopenia (9)

The number of studies on COVID-19 infection and muscle indices is extremely low in the literature even though sarcopenia and low muscle mass are known to be poor prognostic factors in many diseases. Evaluation of paravertebral muscle indices obtained by CT may help us to better understand the effects of COVID-19 on prognosis. This study aims to find an answer to the question of whether muscle mass measures such as SMA, SMI, SMD, and SMG obtained from thoracic CT scans taken at the time of diagnosis of patients hospitalized for COVID-19 infection are an independent risk factor effective on mortality, NIMV and need for intensive care (IC). Our study is the first in the literature to evaluate the effects of muscle measures obtained from the T12 level on prognosis in adult patients hospitalized with COVID-19 infection to the best of our knowledge.

\section{Materials and methods}

\section{Study population}

Our retrospective study was approved by the Ministry of Health, General Directorate of Health Services of the Republic of Turkey, and Mugla Sitki Kocman University Human Research Ethics Committee. Since our study design was retrospective, written informed consent was not obtained from the patients to participate in the study. COVID-19 patients over 18 years of age who were diagnosed with COVID-19 by RT-PCR test between 20 March 2020 and 10 August 2020, who were hospitalized in the COVID-19 service and intensive care units of MSKU Training and Research Hospital due to high clinical disease severity, and who underwent non-contrast thoracic $\mathrm{CT}$ examination at the time of diagnosis were included in the study. Thoracic CT indications were determined according to the COVID-19 Patient Management Algorithm of the Ministry of Health of the Republic of Turkey.

The patients' age, comorbidity index (CCI) scores, gender, anthropometric criteria such as height, weight, and BMI, laboratory values, development of NIMV and IC need during follow-up, length of hospital stay, and hospital mortality were retrospectively screened through our hospital automation system in our study. NIMV was applied to patients with hypoxemic respiratory failure (partial arterial oxygen pressure $\left(\mathrm{paO}_{2}\right)<60 \mathrm{mmHg}$ ) in the absence of invasive mechanical ventilation (IMV) indications. Patients whose hypoxemia did not improve with NIMV treatment and who needed IMV were treated in the intensive care units. SMA, SMI, SMD, SMG measurements of the paravertebral muscles at the level of the T12vertebra were obtained from the thoracic CT images taken at the time of diagnosis.

\section{CT examination}

Thorax non-contrast CT of all cases was performed with a multidetector CT scanner reserved for patients with suspected COVID-19 (Toshiba Asteion). Approved disinfectants were used in accordance with the procedures of our hospital for disinfection of the CT room and the surfaces in contact with the patient. Thorax CT parameters were $4 \times 1.5 \mathrm{~mm}$ section collimation, $3 \mathrm{~mm}$ section thickness, the field of view, $120 \mathrm{kV}$ tube voltage, tube current 200 $\mathrm{mA} / \mathrm{sec}$, and matrix $512 \times 512$.

\section{Measurement methods}

Muscle index measurements of the cases were performed at the level of upper end-plates of the T12 vertebra. 3D SLICER was used for muscle index measurement (http://www.slicer.org, Surgical Planning Laboratory, Harvard University, Boston, MA, USA, version 4.10.2). Thorax CT examination of the case was imported into

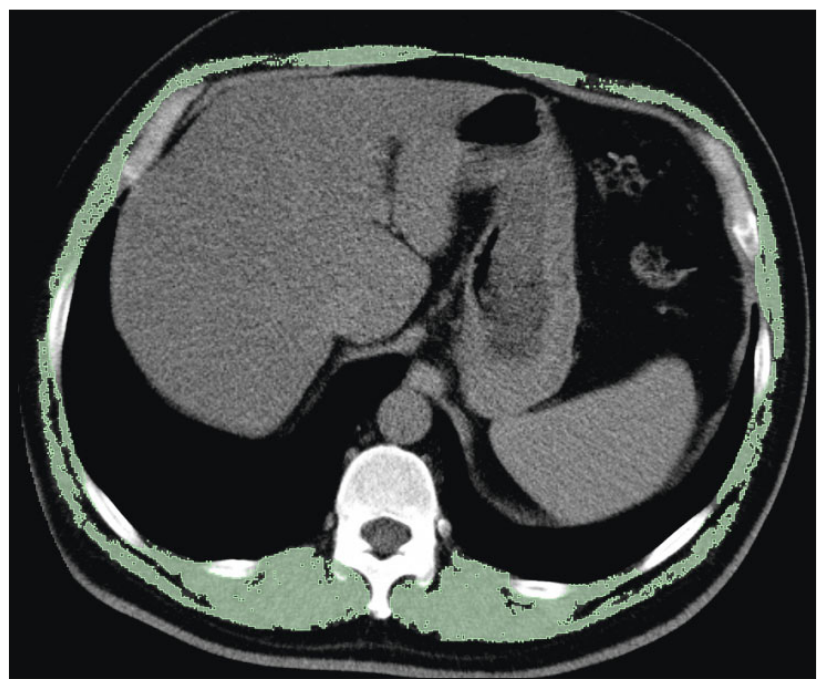

Fig. 1. Measurement of skeletal muscle area at the level of the $12^{\text {th }}$ thoracic vertebra on chest computed tomography image in a 52-yearold man. 
a 3D slicer for further examination. $1.5 \mathrm{~mm}$ axial reconstructions were used for measurements. A threshold containing muscle tissue was determined using the segment editor module (range 0-150). Subsequently, only the muscle tissue was stained manually with the paint effect using the determined threshold mask (Fig. 1). The areas of the muscles (SMA, $\mathrm{cm}^{2}$ ) were measured for each level using the radiomics module after painting (Fig. 1). SMI was obtained for each patient using the calculation formula ((skeletal muscle area- $\left.\mathrm{cm}^{2}\right) /\left(\right.$ patient height- $\left.\mathrm{m}^{2}\right)$ ). The density (SMD) of the muscles at all levels was measured in Hounsfield and obtained by averaging the HU radiodensity for the total cross-sectional skeletal muscle. SMG measurement was performed to evaluate both SMI and SMD by integrating. SMG was obtained using the SMI $\times$ SMD formula.
No significant difference was observed in terms of BMI values according to mortality status $(\mathrm{p}=0.163)$ whereas T12SMD values were significantly lower in the mortality group compared to the non-mortality group in our study $(p=0.025)$. In addition, ferritin $(p=0.013)$ and $D$-dimer values $(p=0.007)$ were found to be statistically significantly higher in the mortality group compared to the non-mortality group (Table 2$)$.

$\mathrm{CCI}(\mathrm{p}=0.036), \mathrm{CRP}(\mathrm{p}=0.009)$, procalcitonin $(\mathrm{p}=0.012)$, ferritin $(p<0.001)$, and D-dimer values $(p=0.039)$ were found to be statistically significantly higher in the group showing NIMV need compared to the group not showing NIMV need. No statistically significant difference was observed between patients with and without NIMV need in terms of BMI values (p: 0.448) whereas

\section{Statistical analysis}

Data were analyzed using SPSS software version 22.0 for Windows (SPSS Inc, Chicago, Illinois, USA). The suitability of the data for normal distribution was evaluated by the Kolmogorov-Smirnov Test. Comparisons of numerical variables between two independent groups were performed by Independent samples t-test when normal distribution condition was met and Mann -Whitney U test when not met. Correlation coefficient and statistical significance were calculated by Pearson test for the relationships between normally distributed variables, and correlation coefficient and statistical significance were calculated by Spearman's test for the relationships between variables at least one of which was not normally distributed. Linear regression analysis was performed to determine independent risk factors affecting mortality, NIMV, and the need for IC. ROC curve analysis was performed to determine the cut-off value in terms of showing NIMV and intensive care need of paravertebral muscle indices. Statistical significance was interpreted using the "p" value in the obtained data. p-value $<0.05$ was considered statistically significant.

\section{Results}

A total of 68 people, 29 (42.6\%) female and $39(57.4 \%)$ male, were included in the study. The mean age of the cases was 48.5 $( \pm 18.1)$. The need for NIMV developed in $15.9 \%$ of the cases during follow-up whereas $19 \%$ showed the need for intensive care. 5 patients $(7.6 \%)$ died during their followup. Descriptive characteristics of the study population are presented in Table 1 .
Tab. 1. Descriptive characteristics of the study population by gender.

\begin{tabular}{|c|c|c|c|c|}
\hline Characteristic & $\begin{array}{c}\text { Total population } \\
(\mathrm{n}=68) \\
\text { Mean }( \pm \mathrm{sd})\end{array}$ & $\begin{array}{c}\text { Male } \\
(\mathrm{n}=39) \\
\text { Mean }( \pm \text { sd })\end{array}$ & $\begin{array}{c}\text { Female } \\
(\mathrm{n}=29) \\
\text { Mean }( \pm \mathrm{sd})\end{array}$ & $\mathrm{p}$ \\
\hline Age & $48.5( \pm 18.1)$ & $47.7( \pm 16.6)$ & $49.6( \pm 20.2)$ & 0.664 \\
\hline BMI & $27.5( \pm 4.5)$ & $27.4( \pm 3.5)$ & $27.6( \pm 5.5)$ & 0.878 \\
\hline T12SMA & $80.1( \pm 31.7)$ & $98.5( \pm 29.0)$ & $56.9( \pm 15.7)$ & $<.001$ \\
\hline T12SMI & $40.7( \pm 11.9)$ & $47.9( \pm 11.5)$ & $33.4( \pm 7.1)$ & $<.001$ \\
\hline SMD & $38.4( \pm 14.6)$ & $38.7( \pm 4.8)$ & $37.9( \pm 4.6)$ & 0.834 \\
\hline T12SMG & $1579.1( \pm 840.9)$ & $1795.8( \pm 965.0)$ & $1335.2( \pm 606.3)$ & 0.045 \\
\hline $\mathrm{Ln}$ & $2.1( \pm 4.6)$ & $2.5( \pm 6.2)$ & $1.5( \pm 0.6)$ & 0.369 \\
\hline $\mathrm{Neu}$ & $4.4( \pm 3.9)$ & $4.5( \pm 2.7)$ & $4.2( \pm 4.9)$ & 0.684 \\
\hline PLT & $213.9( \pm 70.8)$ & $212.4( \pm 66.4)$ & $215.8( \pm 77.0)$ & 0.850 \\
\hline UA & $5.6( \pm 2.4)$ & $5.3( \pm 2.0)$ & $5.9( \pm 2.9)$ & 0.606 \\
\hline $\mathrm{Ca}$ & $8.8( \pm 0.8)$ & $8.9( \pm 0.7)$ & $8.6( \pm 0.7)$ & 0.113 \\
\hline $\mathrm{Na}$ & $137.6( \pm 3.7)$ & $138.1( \pm 4.3)$ & $136.9( \pm 2.8)$ & 0.208 \\
\hline Alb & $4.0( \pm 0.6)$ & $4.9( \pm 0.6)$ & $3.9( \pm 0.5)$ & 0.268 \\
\hline AST & $31.3( \pm 31.8)$ & $36.4( \pm 40.5)$ & $24.9( \pm 13.6)$ & 0.151 \\
\hline \multirow[t]{2}{*}{ ALT } & $27.9( \pm 20.8)$ & $33.7( \pm 19.9)$ & $19.5( \pm 19.6)$ & 0.005 \\
\hline & Median(min-max) & $\operatorname{Median}(\min -\max )$ & $\operatorname{Median}(\min -\max )$ & \\
\hline $\mathrm{CCI}^{*}$ & $2.0(0-12)$ & $2.0(0-12)$ & $1.5(0-9)$ & 0.781 \\
\hline WBC* & $5.4(1.2-31.4)$ & $6.1(1.2-14.4)$ & $4.9(2.7-31.4)$ & 0.099 \\
\hline $\mathrm{Mn*}$ & $0.6(0.2-1.3)$ & $0.6(0.3-1.2)$ & $0.5(0.18-1.3)$ & 0.122 \\
\hline Glu* & $108(70-210)$ & $111(83-210)$ & $102(70-157)$ & 0.109 \\
\hline Cre* & $0.9(0.5-10.7)$ & $1.0(0.7-10.7)$ & $0.8(0.5-5.5)$ & $<0.001$ \\
\hline $\mathrm{LDH}^{*}$ & $203(26-696)$ & $205(26-696)$ & $198(110-610)$ & 0.745 \\
\hline $\mathrm{CPK}^{*}$ & $89(17-4667)$ & $117(18-4667)$ & $67(17-485)$ & 0.003 \\
\hline T.Bil* & $0.4(0.1-6.3)$ & $0.4(0.1-0.7)$ & $0.4(0.1-6.3)$ & 0.921 \\
\hline D-Dimer* & $264(150-8266)$ & $264(150-8266)$ & $264(150-7705)$ & 0.879 \\
\hline CRP* & $12.9(0.6-531)$ & $16.5(1-531)$ & $8.18(0.6-274)$ & 0.135 \\
\hline Procal* & $0.07(0.01-92.9)$ & $0.07(0.0-92.9)$ & $0.06(0.01-5.8)$ & 0.125 \\
\hline Ferritine* & 188.3(8.5-2942) & 335 (59.9-2942) & $79(8.5-989)$ & $<0.001$ \\
\hline Day* & $7(1-87)$ & $7(1-26)$ & $6(1-87)$ & 0.368 \\
\hline
\end{tabular}

Variables with normal distribution were specified as mean ( \pm sd deviation), and Independent samples T test was applied for these variables. *Variables with non-normal distribution are specified as median (min-max), and Mann Whitney $\mathrm{U}$ test was used for these variables.

CCI - Charlson Comorbidity Index, WBC - White blood cell, Mn - monocyte, Glu - glucose, Cre - creatinine, LDH - lactate dehydrogenase, CPK - creatine phosphokinase, T.Bil - total bilirubin, CRP - C-reactive protein, Procal - procalcitonin, Day - length of stay in the hospital. BMI - Body mass index, - :12. Thoracic Vertebra Skeletal muscle area, T12SMI - 12. Thoracic Vertebra Skeletal Muscle Index, SMD - Skeletal muscle density, T12SMG - 12. Thoracic Vertebra Skeletal muscle gauge, Ln - Lymphocyte, Neu - neutrophil, Mn - monocyte, PLT - platelet, UA - uric acid, Ca - calcium, Na - sodium, Alb - albumin, AST - aspartate aminotransferase, ALT - alanine aminotransferase. 
T12SMD values were significantly lower in the group with NIMV need compared to the other group $(p=0.048)$ (Tab. 2).

CCI $(p=0.036), \operatorname{CRP}(p=0.002)$, procalcitonin $(p=0.039)$, ferritin $(p<0.001)$, and D-dimer values $(p=0.005)$ were found to be statistically significantly higher in patients in need of IC compared to the group without IC. No statistically significant difference was observed between the patients with and without intensive care needs in terms of BMI values $(\mathrm{p}=0.749)$ whereas T12SMD values were significantly lower in the IC group compared to the other group ( $p=0.029)$ (Tab. 2).

The adjusted R-squared value of the regression model created with age, BMI, CCI, glucose, creatinine, total bilirubin, alanine aminotransferase (ALT), albumin, lactate dehydrogenase (LDH), C reactive protein (CRP), procalcitonin, ferritin, D-dimer, T12SMA, T12SMI, T12SMG, and SMD variables was observed to be 93 in order to determine independent risk factors affecting mortality in individuals with COVID-19 infection. $93 \%$ of the variables affecting mortality could be explained by this obtained model, which supported that the model was good. T12SMD $(\beta=-0.307 ; \mathrm{p}=0.008)$, albumin $(\beta=-0.073 ; p=0.011), \operatorname{CRP}(\beta=0.781 ; p=0.001)$, and $D$-dimer values $(\beta=0.489 ; p=0.021)$ were shown to be independent risk factors affecting mortality in patients hospitalized due to COVID-19 infection in linear regression analysis (Table 3). It was shown that albumin, procalcitonin values and T12SMD are the most important independent risk factors for mortality in the multiple linear regression analysis (Tab. 3).

The adjusted R-squared value of the regression model created with age, BMI, CCI, glucose, creatinine, total bilirubin, ALT, albumin, LDH, CRP, procalcitonin, ferritin, D-dimer, T12SMA, T12SMI, T12SMG, and SMD variables was observed to be 78 in order to determine independent risk factors affecting the need for NIMV in individuals with COVID-19 infection. $78 \%$ of the variables affecting NIMV could be explained by this obtained model, which supported that the model was good. Age $(\beta=0.541$; $p=0.041)$, T12SMD $(\beta=-0.733 ; p=0.001)$, glucose $(\beta=0.335$; $\mathrm{p}=0.02)$, and ALT values $(\beta=0.349 ; \mathrm{p}=0.017)$ were shown to be independent risk factors affecting the need for NIMV in patients hospitalized due to COVID-19 infection in linear regression analysis (Tab. 4). It was shown that glucose and T12SMD are the most important independent risk factors for NIMV need in the multiple linear regression analysis (Tab. 4).

The adjusted R-squared value of the regression model created with age, BMI, CCI, glucose, creatinine, total bilirubin, ALT, albumin, LDH, CRP, procalcitonin, ferritin, D-dimer, T12SMA, T12SMI, T12SMG, and SMD variables was observed to be 94 in order to determine independent risk factors affecting the need for IC in individuals with COVID-19 infection. $94 \%$ of the variables affecting the need for IC could be explained by this obtained model, which supported that the model was good. Age $(\beta=0.820 ; \mathrm{p}=0.001)$, CCI $(\beta=0.626 ; p=0.001)$, T12SMD $(\beta=-0.912 ; p=0.001)$, creatinine $(\beta=0.664 ; p=0.02), \mathrm{LDH}(\beta=0.355 ; p=0.033), \mathrm{CRP}$ $(\beta=1.619 ; p=0.001)$, and $D$-dimer values $(\beta=0.833 ; p=0.001)$ were shown to be independent risk factors affecting the need for IC in patients hospitalized due to COVID-19 infection in linear regression analysis (Tab. 5). It was shown that creatinine, D-Dimer levels 
Tab. 3. Evaluation of independent risk factors for mortality by logistic regression analysis.

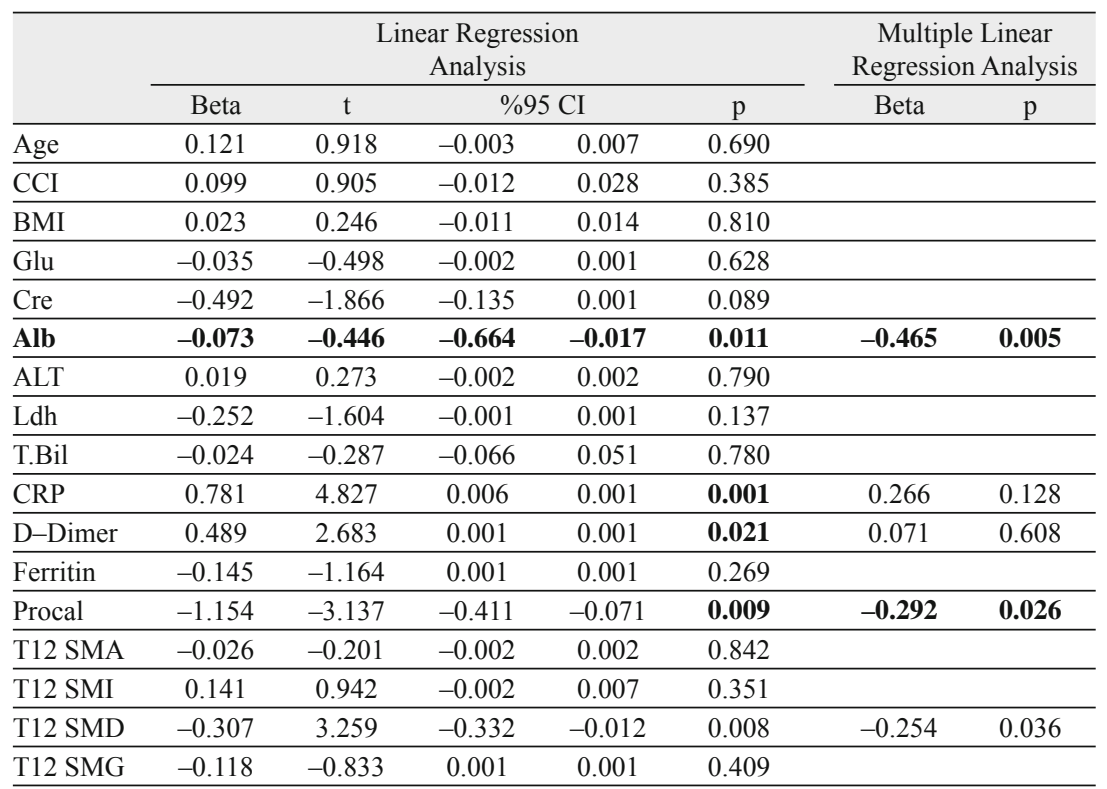

CCI - Charlson Comorbidity Index, BMI - Body mass index, T12SMA - 12. Thoracic Vertebra Skeletal muscle area, T12SMI - 12. Thoracic Vertebra Skeletal muscle Index, SMD - Skeletal muscle density, T12SMG:12. Thoracic Vertebra Skeletal muscle gauge, Glu - glucose, Cre - creatinine, Alb - albumin, ALT - alanine aminotransferase, LDH - lactate dehydrogenase, T.Bil - total bilirubin, CRP - C-reactive protein, Procal - procalcitonin

Tab. 4. Evaluation of independent risk factors for non-invasive mechanic ventilation needs by logistic regression analysis.

\begin{tabular}{|c|c|c|c|c|c|c|c|}
\hline \multirow{3}{*}{$\begin{array}{l}\text { Age } \\
\end{array}$} & \multicolumn{5}{|c|}{$\begin{array}{c}\text { Linear Regression } \\
\text { Analysis } \\
\end{array}$} & \multicolumn{2}{|c|}{$\begin{array}{c}\text { MultipleLinear } \\
\text { Regression Analysis }\end{array}$} \\
\hline & \multirow{2}{*}{$\begin{array}{c}\text { Beta } \\
0.541\end{array}$} & \multirow{2}{*}{$\frac{\mathrm{t}}{2.313}$} & \multicolumn{2}{|c|}{$\% 95 \mathrm{CI}$} & \multirow{2}{*}{$\begin{array}{c}\mathrm{p} \\
0.041\end{array}$} & \multirow{2}{*}{$\begin{array}{c}\text { Beta } \\
0.083 \\
\end{array}$} & \multirow{2}{*}{$\begin{array}{c}\mathrm{p} \\
0.619\end{array}$} \\
\hline & & & 0.001 & 0.030 & & & \\
\hline$\overline{\mathrm{CCI}}$ & 0.417 & 2.140 & -0.002 & 0.109 & 0.059 & & \\
\hline BMI & 0.128 & 0.765 & -0.023 & 0.047 & 0.460 & & \\
\hline Glu & 0.335 & 2.720 & 0.001 & 0.009 & 0.020 & 0.274 & 0.042 \\
\hline Cre & 0.659 & 1.409 & -0.074 & 0.336 & 0.187 & & \\
\hline Alb & 0.184 & 0.633 & -0.028 & 0.050 & 0.540 & & \\
\hline ALT & 0.349 & 2.806 & 0.002 & 0.013 & 0.017 & 0.123 & 0.333 \\
\hline Ldh & 0.245 & 0.879 & -0.001 & 0.003 & 0.398 & & \\
\hline T.Bil & 0.104 & 0.695 & -0.113 & 0.217 & 0.502 & & \\
\hline CRP & -0.829 & -1.266 & -0.009 & 0.002 & 0.232 & & \\
\hline DDimer & 0.699 & 2.163 & 0.001 & 0.001 & 0.053 & & \\
\hline Ferritin & 0.137 & 0.618 & 0.001 & 0.002 & 0.549 & & \\
\hline Procal & 0.067 & 0.103 & -0.454 & 0.498 & 0.920 & & \\
\hline T12 SMA & 0.080 & 0.602 & -0.002 & 0.004 & 0.482 & & \\
\hline T12 SMI & -0.009 & -0.060 & -0.008 & 0.008 & 0.953 & & \\
\hline T12 SMD & -0.733 & -4.380 & -0.042 & -0.014 & 0.001 & -0.322 & 0.047 \\
\hline T12 SMG & -0.187 & -1.307 & 0.001 & 0.001 & 0.198 & & \\
\hline
\end{tabular}

NIMV - Non-invasive mechanic ventilation, CCI - Charlson Comorbidity Index, BMI - Body mass index, T12SMA - 12. Thoracic Vertebra Skeletal muscle area, T12SMI - 12. Thoracic Vertebra Skeletal Muscle Index, SMD - Skeletal muscle density, T12SMG - 12. Thoracic Vertebra Skeletal muscle gauge, Glu - glucose, Cre creatinine, Alb - albumin, ALT - alanine aminotransferase, LDH - lactate dehydrogenase, T.Bil - total bilirubin, $\mathrm{CRP}-\mathrm{C}$ reactive protein, Procal - procalcitonin

and T12SMD are the most important independent risk factors for the need for IC in the multiple linear regression analysis (Tab. 5).

12th T12SMD cut-off value was determined to be 40.134 with $70.4 \%$ sensitivity and $66.7 \%$ specificity $(p=0.006$ area under curve (AUC): 0.754 ) as a result of ROC curve analysis conducted to determine the cut-off value of thoracic vertebra SMD values in terms of indicator of NIMV need in COVID-19 cases (Fig. 2). T12SMG cut-off value was determined to be 1320.23 with $73.2 \%$ sensitivity and $70 \%$ specificity $(\mathrm{p}=0.018$ AUC: 0.744$)$ as a result of ROC curve analysis conducted to determine the cut-off value of T12SMG values in COVID-19 cases in terms of indicator of NIMV need (Fig. 2). No statistically significant result was obtained in ROC curve analysis conducted to determine the cut-off value of BMI value in terms of indicator of NIMV need in COVID-19 cases ( $p=0.499$ AUC: 0.576).

\section{Discussion}

T12SMD, which is one of the paravertebral muscle indices at the T12 vertebra level obtained from thoracic CT, has been shown to be significantly associated with various negative outcomes such as mortality, need for NIMV, and need for intensive care independently of BMI index. Our study is the first to evaluate the effects of paravertebral muscle indices at thoracic vertebra level on mortality, NIMV, and need for intensive care in thoracic CT scans of adult patients hospitalized for COVID-19 infection to the best of our knowledge. Moreover, a cut-off value (40.134, $70.4 \%$ sensitivity, $66.7 \%$ specificity, $p=0.006$, AUC: 0.754 ) was determined for T12SMD value as an indicator of NIMV need in our study. Similarly, T12 SMD measurements were significantly lower in patients with NIMV and intensive care needs in accordance with the current results in this study. Our study is the first to show that T12SMD measurements can be used as a marker and an indicator of the need for NIMV in patients hospitalized for COVID-19 infection in this respect. In addition, the results obtained from our study show that information can be obtained about the prognosis of COVID-19 cases by evaluating not only lung tissue but also paravertebral muscle density with T12SMD to evaluate pneumonia and disease severity, and at the same time when thoracic CTs of patients with COVID-19 are evaluated and it can be used as a marker for closer follow-up of cases with low muscle indices in terms of NIMV and need for IC.

There are many studies in the literature on the factors determining the prognosis in COVID-19 patients. In these studies, it 
Tab. 5. Evaluation of independent risk factors for intensive care needs by logistic regression analysis.

\begin{tabular}{|c|c|c|c|c|c|c|c|}
\hline \multirow[b]{3}{*}{ Age } & \multicolumn{5}{|c|}{$\begin{array}{c}\text { Linear Regression } \\
\text { Analysis }\end{array}$} & \multicolumn{2}{|c|}{$\begin{array}{c}\text { Multiple Linear } \\
\text { Regression Analysis }\end{array}$} \\
\hline & \multirow{2}{*}{$\begin{array}{c}\text { Beta } \\
0.820\end{array}$} & \multirow{2}{*}{$\frac{\mathrm{t}}{6.716}$} & \multicolumn{2}{|c|}{$\% 95 \mathrm{CI}$} & \multirow{2}{*}{$\begin{array}{c}\mathrm{p} \\
0.001\end{array}$} & \multirow{2}{*}{$\begin{array}{c}\text { Beta } \\
-0.112\end{array}$} & \multirow{2}{*}{$\begin{array}{c}\mathrm{t} \\
0.553\end{array}$} \\
\hline & & & 0.015 & 0.029 & & & \\
\hline$\overline{\mathrm{CCI}}$ & 0.626 & 6.157 & 0.048 & 0.102 & 0.001 & 0.040 & 0.821 \\
\hline$\overline{\mathrm{BMI}}$ & -0.170 & -1.956 & -0.032 & 0.002 & 0.076 & & \\
\hline$\overline{\text { Glu }}$ & 0.097 & 1.512 & -0.001 & 0.003 & 0.159 & & \\
\hline Cre & 0.664 & 2.722 & 0.024 & 0.223 & 0.020 & 0.356 & 0.001 \\
\hline$\overline{\mathrm{Alb}}$ & 0.161 & 1.061 & -0.010 & 0.028 & 0.312 & & \\
\hline$\overline{\text { ALT }}$ & 0.099 & 1.530 & -0.001 & 0.005 & 0.154 & & \\
\hline Ldh & 0.355 & 2.438 & 0.001 & 0.002 & 0.033 & 0.094 & 0.499 \\
\hline T.Bil & -0.066 & -.840 & -0.111 & 0.050 & 0.419 & & \\
\hline$\overline{\mathrm{CRP}}$ & 1.619 & 4.737 & 0.003 & 0.008 & 0.001 & 0.154 & 0.226 \\
\hline D-Dimer & 0.833 & 4.934 & 0.001 & 0.001 & 0.001 & 0.446 & 0.003 \\
\hline Ferritin & 0.204 & 1.761 & 0.001 & 0.002 & 0.106 & & \\
\hline Procal & 0.591 & 1.734 & -0.049 & 0.415 & 0.111 & & \\
\hline T12 SMA & 0.072 & 0.540 & -0.002 & 0.004 & 0.591 & & \\
\hline T12 SMI & -0.001 & -0.007 & -0.007 & 0.007 & 0.994 & & \\
\hline T12 SMD & -0.912 & -10.446 & -0.039 & -0.026 & 0.001 & -0.274 & 0.044 \\
\hline T12 SMG & -0.196 & -1.370 & 0.001 & 0.001 & 0.177 & & \\
\hline
\end{tabular}

CCI - Charlson Comorbidity Index, BMI - Body mass index, T12SMA - 12. Thoracic Vertebra Skeletal muscle area, T12SMI - 12. Thoracic Vertebra Skeletal Muscle Index, SMD - Skeletal muscle density, T12SMG - 12. Thoracic Vertebra Skeletal muscle gauge, Glu - glucose, Cre - creatinine, Alb - albumin, ALT - alanine aminotransferase, LDH - lactate dehydrogenase, T.Bil - total bilirubin, CRP - C-reactive protein, Procal - procalcitonin

has been reported that male gender and advanced age ( $>65$ years), as well as cardiovascular diseases such as heart failure, diabetes mellitus, malignancies, and cerebrovascular diseases, are strong and independent determinants of mortality (10-11). There are also studies in the literature examining the prognosis of the disease by evaluating thoracic CTs of COVID-19 patients (12). Patients with high pneumonia severity score (PSS) were shown to have a worse prognosis in the study conducted by Chung et al (13). However, the number of studies evaluating the effect of various muscle indices obtained by thoracic $\mathrm{CT}$ on the prognosis of patients hospitalized for COVID-19 infection is extremely limited to our knowledge. Ufuk $F$ et al showed that pneumonia severity score (PSS), pectoralis muscle area, and index values in thoracic CT were significantly associated with various negative outcomes such as intubation, long-term hospitalization, and death in $\mathrm{CO}$ VID-19 patients who underwent thoracic CT at hospitalization (14). The decrease in pectoralis muscle area (PMA) and gender-specific PMA values, one of the strong predictors of sarcopenia, is also known to be associated with malignant prognosis in diseases such as lung cancer, chronic obstructive pulmonary disease, and idiopathic pulmonary fibrosis (15-16).

T12SMD values have been shown to be associated with various negative outcomes such as mortality, need for NIMV, and need for intensive care independently of BMI index, whereas no relationship has been shown between BMI value and mortality, need for NIMV, and need for IC. These results support the opinion that paravertebral muscle index measurements performed to evaluate sarcopenia and
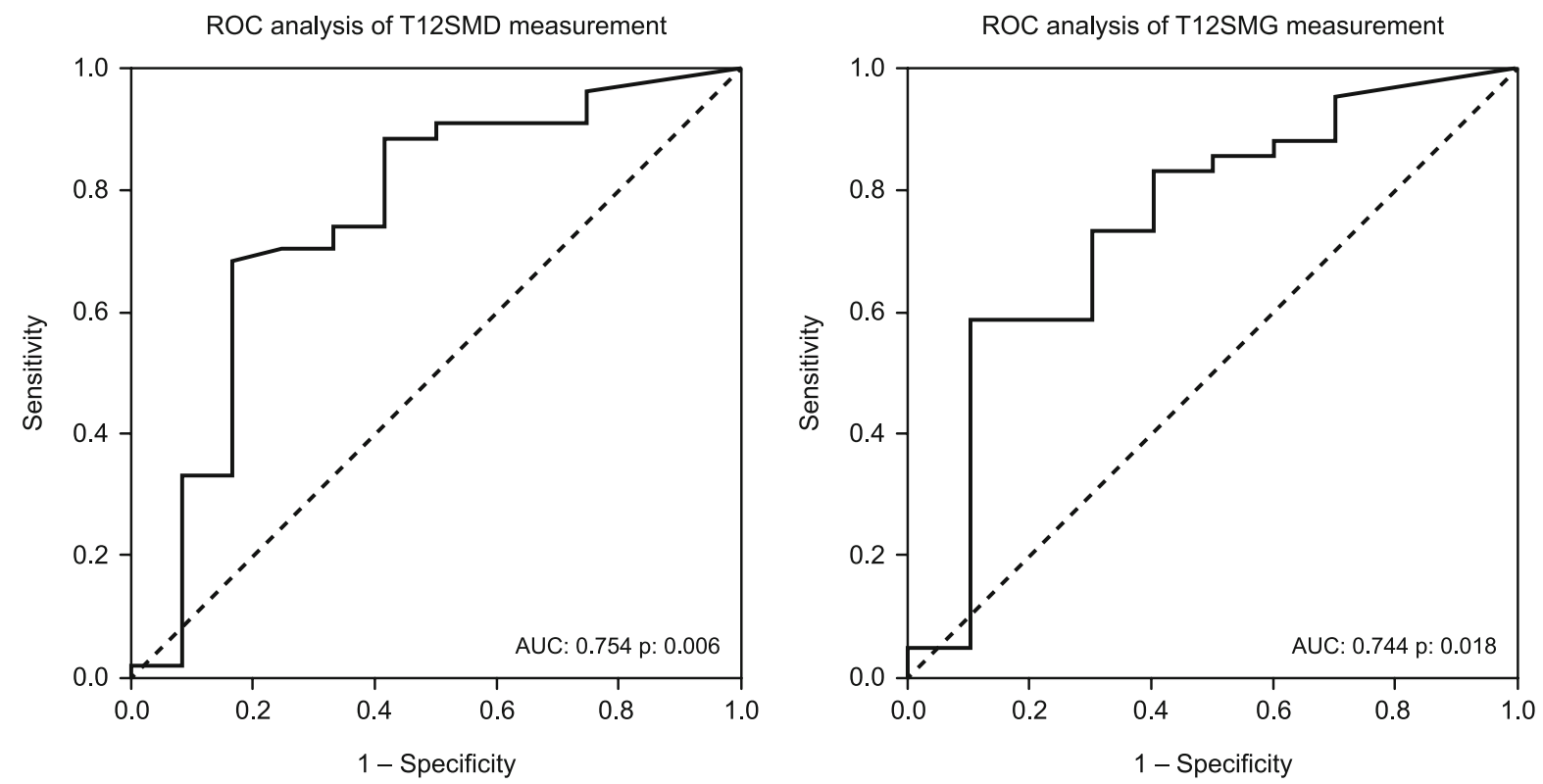

Fig. 2. ROC analysis of $12^{\text {th }}$ thoracic vertebra skeletal muscle density and $12^{\text {th }}$ thoracic vertebra skeletal muscle gauge measurements as an indicator of non-invasive mechanic ventilation requirement. 
muscle mass are a more reliable method than BMI. It is known that BMI may misclassify patient phenotypes because it does not consider body composition (17). In fact, weight conceals the fat mass and water imbalances inside, as it does not represent muscle mass sufficiently. For these reasons, BMI is an inadequate criterion in distinguishing muscle and fat tissue. Low BMI may also mask excessive adiposity whereas high BMI may mask sarcopenia. Therefore, evaluation of body composition criteria that can distinguish body fat tissue distribution, muscle quantity, and quality may be more useful in evaluating muscle mass and sarcopenia. In addition, it is known that this situation may cause dangerous paradoxes and may change the perception of muscle mass by clinicians considering that obesity is a common clinical condition in COVID-19 patients (18).

Quantity (SMI) and density (SMD) measurements of the muscles at the paravertebral level are usually performed to determine muscle mass in CTs. However, the density and quantity of the evaluated muscles do not always show a positive correlation. In other words, muscle density may be low in an individual with a high quantity of muscle whereas muscle density may be high in an individual with a low quantity of muscle. Therefore, it has been stated that the use of a mathematical combination showing both muscle quantity and density may give more meaningful results in terms of predicting muscle mass. Weinberg et al. suggested for the first time that SMG measurement obtained by multiplying SMI and SMD measurements can be used as a new marker in the differentiation of sarcopenia. Studies have also shown that SMG measurement alone with aging gives more reliable results than SMD or SMI $(9,19)$.

No statistically significant relationship was found between T12SMG values obtained in thoracic CT and mortality, NIMV, and the need for IC in our study. However, a cut-off value of T12SMG values was determined to be 1320.2 (73.2 \% sensitivity, $70 \%$ specificity, $p=0.018$ AUC: 0.744 ) as a marker of NIMV need in ROC analysis conducted. These results are important in that they show that T12SMD and T12SMG, one of the paravertebral muscle indices at the T12 level, can be used as a marker to show whether the need for NIMV will develop during the follow-up of patients hospitalized for COVID-19 infection.

The mortality rate is known to be high in elderly patients and in patients with comorbidities such as diabetes even though some COVID-19 patients are asymptomatic or mildly symptomatic (20). It was shown in a study conducted in Italy that $0.8 \%$ of the cases who died due to COVID-19 had no comorbidities whereas $48.5 \%$ of the cases had 3 or more underlying diseases, $25.6 \%$ had 2 or more underlying diseases, and the presence of these comorbidities may have increased the risk of death independent of COVID-19 infection (21).

Many scoring systems have been developed to predict morbidity and mortality since comorbidities accompanying individuals have significant effects on their survival and prognosis. CCI is one of the widely used scoring systems due to its easy and rapid application as well as its success in predicting mortality (22). Many studies in the literature have shown that comorbidities accompanying COVID-19 are one of the independent predictors of in-hospital mortality (20). Christensen et al. showed that a CCI score above 0 was associated with an increase in the clinical course of severe COVID-19 and the risk of death (23). Similarly Gong et al. showed a strong negative correlation between SMI and CCI index in their study involving hospitalized elderly patients (24). CCI scores of patients with NIMV and intensive care need were significantly higher than those without, and CCI value was found to be an independent risk factor affecting the need for NIMV and the need for IC. A strong negative correlation was found between T12 SMA ( $\mathrm{r}=-0.304, \mathrm{p}<0.05)$, SMD $(\mathrm{r}=-0.453, \mathrm{p}<0.01)$, and T12SMG $(r=-0.395, p<0.01)$, which are paravertebral muscle criteria obtained by CT.

The retrospective design of the study, the inability to perform multicenter tomography, the study population being from a single geographical region, the fact that thorax CT was performed only in patients with CT indication according to the guidance of the Ministry of Health of the Republic of Turkey, and thoracic computed tomography could not be performed in all COVID-19 patients, the presence of sarcopenia could not be evaluated by anthropometric measurements (calf circumference, etc.), and other quantitative tests (grip strength measurements, DEXA etc.) and the relatively small sample size were accepted as the limitations of our study. However, our study is important in that it is the first to investigate mortality, NIMV, and the need for IC of T12SMD and T12SMG, one of the T12 muscle indices in thoracic CT scans in adult COVID-19 patients to the best of our knowledge. In addition; age, CCI score, CRP, procalcitonin, D-dimer, glucose, creatinine, ALT, albumin, and LDH values were shown to be independent predictors for many negative results together with T12SMD and T12SMG in our study.

In conclusion, T12SMD and T12SMG, two of the T12 paravertebral muscle indices, are significantly associated with various adverse outcomes in adult COVID-19 patients and provide clinicians with important information about the prognosis of the cases. These parameters which can be easily evaluated in thoracic CT images of COVID-19 patients will be very useful in routine clinical practice since they do not require additional examination. Evaluating not only lung tissue but also paravertebral muscle mass and density with T12SMD and T12SMG to evaluate pneumonia and disease severity when thoracic CTs of patients with COVID-19 are evaluated will provide clinicians with very valuable information about whether cases with low muscle indices will need NIMV and IC or not. The evaluation of these indices will contribute to the early recognition of sarcopenia in cases with normal BMI scores and without any pathology considering that obesity is a common clinical condition in COVID-19 patients and this situation may change the perception of muscle mass by clinicians (25). Thus, we think that closer and careful follow-up of cases with low muscle indices regardless of BMI scores may contribute to the reduction of the risk of COVID-related complications and mortality. In this context, we think that T12SMD and T12SMG indices can be used as new markers showing mortality, and the need for NIMV and IC during the diagnosis and followup of patients with COVID-19. 


\section{References}

1. Catharine IP, Hilary DM, Anthony SF. Coronavirus InfectionsMore Than Just the Common Cold. JAMA 2020; 323 (8): 707-708. DOI: 10.1001/jama.2020.0757.

2. Xu Z, Shi L, Wang Y, Zhang J, Lei Huang L et al. Pathological findings of COVID-19 associated with acute respiratory distress syndrome. Lancet Respir Med 2020; 8 (4): 420-422. DOI: 10.1016/S2213-2600 (20)30076-X.

3. Li Y, Xia L. Coronavirus Disease 2019 (COVID-19) - Role of Chest CT in Diagnosis and Management. Am J Roentgenol 2020; 214 (6): 12801286.

4. Cruz-Jentoft AJ, Bahat G, Bauer J, Boirie $Y$, Bruyère $O$ et al. Sarcopenia - revised European consensus on definition and diagnosis. Age Ageing 2019; 48 (1): 16-31. DOI: 10.1093/ageing/afy169.

5. Gilligan LA, Towbin AJ, Dillman JR, Somasundaram E, Trout AT et al. Quantification of skeletal muscle mass: sarcopenia as a marker of overall health in children and adults. Pediatr Radiol 2020; 50 (4): 455-464. DOI: $10.1007 / \mathrm{s} 00247-019-04562-7$.

6. Derstine BA, Holcombe SA, Goulson RL, Ross BE, Wang NC et al. Quantifying Sarcopenia Reference Values Using Lumbar and Thoracic Muscle Areas in a Healthy Population. J Nutr Health Aging 2017; 21 (10): 180-185. DOI: 10.1007/s12603-017-0983-3.

7. Walowski CO, Braun W, Maisch MJ, Jensen B, Peine S et al. Reference Values for Skeletal Muscle Mass-Current Concepts and Methodological Considerations. Nutrients 2020; 12 (3): 755. DOI: 10.3390/nu12030755.

8. Derstine BA, Holcombe SA, Ross BE, Wang NC, Su GL et al. Skeletal muscle cutoff values for sarcopenia diagnosis using T10 to L5 measurements in a healthy US population. Sci Rep 2018; 8 (1): 11369. DOI: 10.1038/s41598-018-29825-5.

9. Weinberg M, Shachar S, Deal A, Williams G, Nyrop K et al. Characterization of skeletal muscle and body mass indices in younger and older women with stage II and III breast cancer. J Am Geriatr Soc 2016; (Suppl): S86.

10. Du RH, Liang LR, Yang CQ, Wang W, Cao TZ et al. Predictors of mortality forpatientsith COVID-19 pneumonia caused by SARS-CoV-2 - a prospective cohort study. Eur Respir J 2020; 55 (5): 2000524. DOI: 10.1183/13993003.00524-2020.

11. Petrilli CM, Jones SA, Yang J, Rajagopalan H, O'Donnell L et al. Factors associated with hospital admission and critical illness among 5279 people with coronavirus disease 2019 in New York City - prospective cohort study. BMJ 2020; 369: m1966. DOI: 0.1136/bmj.m1966.

12. Li K, Fang Y, Li W, Pan C, Qin P et al. CT image visual quantitative evaluation and clinical classification of coronavirus disease (COVID-19). Eur Radiol 2020; 30 (8): 4407-4416. DOI: 10.1007/s00330-020-06817-6.

13. Chung M, Bernheim A, Mei $X$, Zhang N, Huang $M$ et al. CT imaging features of 2019 novel coronavirus (2019-nCoV). Radiology 2020; 295 (1): 202-207. DOI: 10.1148/radiol.2020200230.
14. Ufuk F, Demirci M, Sagtas E, Akbudak IH, Ugurlu E et al. The prognostic value of pneumonia severity score and pectoralis muscle Area on chest CT in adult COVID-19 patients. Eur J Radiol 2020; 131: 109271. DOI: 10.1016/j.ejrad.2020.109271.

15. Moon SW, Choi JS, Lee SH, Jung KS, Jung JY et al. Thoracic skeletal muscle quantification - low muscle mass is related with worse prognosis in idiopathic pulmonary fibrosis patients. Respir Res 2019; 20 (1): 35. DOI: 10.1186/s12931-019-1001-6.

16. Kim YS, Kim EY, Kang SM, Ahn HK, Kim HS. Single cross-sectional area of pectoralis muscle by computed tomography - correlation with bioelectrical impedance based skeletal muscle mass in healthy subjects. Clin Physiol Funct Imaging 2017; 37 (5): 507-511. DOI: 10.1111/ cpf.12333.

17. Romano L, Marchetti M, Gualtieri P, RenzoLD, Belcastro $M$ et al. Effects of a Personalized VLCKD on Body Composition and Resting Energy Expenditure in the Reversal of Diabetes to Prevent Complications. Nutrients 2019; 11 (7): 1526. DOI: 10.3390/nu11071526.

18. Zheng KI, Gao F, Wang XB, Sun QF, Pan KH et al. Letter to the Editor - Obesity as a risk factor for greater severity of COVID-19 in patients with metabolic associated fatty liver disease. Metabolism 2020; 108 : 154244. DOI: 10.1016/j.metabol.2020.154244.

19. Williams GR, Deal AM, Muss HB, Weinberg MS, Sanoff HK et al. Skeletal muscle measures and physical function in older adults with cancer-sarcopenia or myopenia? Oncotarget 2017; 8 (20): 33658-33665. DOI: 10.18632 /oncotarget.16866.

20. Imam Z, Odish F, Gill I, O’Connor D, Armstrong J et al. Older age and comorbidity are independent mortality predictors in a large cohort of 1305 COVID-19 patients in Michigan, United States. J Intern Med 2020; 288 (4): 469-476. DOI: 10.1111/joim. 13119.

21. Onder G, Rezza G, Brusaferro S. Case-fatality rate and characteristics of patients dying in relation to COVID-19 in Italy. JAMA 2020; 323 (18): 1775-1776. DOI: 10.1001/jama.2020.4683.

22. Charlson ME, Pompei P, Ales KL, MacKenzie CR. A new method of classifying prognostic comorbidity in longitudinal studies - development and validation. J Chronic Dis. 1987; 40 (5): 373-383. DOI: 10.1016/00219681 (87)90171-8.

23. Christensen DM , Strange JA, Gislason G, Pedersen CT, Gerds T et al. Charlson Comorbidity Index Score and Risk of Severe Outcome and Death in Danish COVID-19 Patients. J Gen Intern Med 2020; 35 (9): 2801-2803. DOI: 10.1007/s11606-020-05991-z.

24. Gong G, Wan W, Zhang X, Liu Y, Liu X et al. Correlation between the Charlson comorbidity index and skeletal muscle mass/physical performance in hospitalized older people potentially suffering from sarcopenia. BMC Geriatr 2019; 19 (1): 367. DOI: 10.1186/s12877-019-1395-5.

25. Zheng KI, Gao F, Wang XB, Sun QF, Pan KH et al. Letter to the Editor - Obesity as a risk factor for greater severity of COVID-19 in patients with metabolic associated fatty liver disease. Metabolism 2020; 108 : 108 - 154244. DOI: 10.1016/j.metabol.2020.154244. 